\title{
STUDY OF THE MAGNETIZATION PROCESSES IN FeB-BASED AMORPHOUS RIBBONS
}

\author{
Jozef KOVÁČ $\check{~}^{*}$ Ladislav NOVÁK ${ }^{* *}$ \\ * Inst. of Exp. Physics, Slovak Academy of Sciences, Watsonova 47, 04001 Košice, Slovak Republic, e-mail: jkovac@saske.sk \\ ** Department of Physics, Faculty of Electrical Engineering and Informatics, Technical University of Košice, Letná 9, 04200 Košice, \\ Slovak Republic
}

\begin{abstract}
The magnetization processes in amorphous ferromagnetic alloys were studied by the hyperbolic model of hysteresis. The measured hysteresis data were used for separation of the domain wall movement, domain rotation and domain wall annihilation and nucleation process during decreasing of the excitation magnetic field. The external applied tensile stress was used as a parameter. Our results show that the process of the domain rotation is the most sensitive for the applied stress. The corresponding magnetization energy decreases with increasing of the mechanical stress for all our studied samples with the positive magnetostriction coefficient.
\end{abstract}

Keywords: amorphous ferromagnetic alloys, magnetization process, modeling of hysteresis loop

\section{INTRODUCTION}

The using of soft ferromagnetic materials (amorphous or nanocrystalline) in industrial praxis (as cores for transformers, sensors, and so on) needs the knowledge about the mechanism of magnetization and demagnetization processes. The hysteresis models [1-4] published so far are based on the separate domain wall movement with domain wall pinning coercivity and domain rotation processes working against an effective anisotropy field. This can be accepted in crystalline materials but for amorphous and nanocrystalline materials these two processes are overlapping especially in the low and intermediate magnetic fields. Such an overlapping model is proposed in [5,6] for the splitting of the measured hysteresis loop into two contributions [5] domain wall movement (DWM) and domain rotation (DR) - and into three contributions [6] adding a third contribution - domain wall annihilation and nucleation (DWAN) to the above mentioned DWM and DR processes. We have tried to use this overlapping model for study of the magnetization processes in FeB-based amorphous ribbons magnetized under applied tensile stress.

\section{EXPERIMENTAL}

The initial "hyperbolic model" [3, 7] was further developed in [5, 6, 8, 9]. For the testing of this model we have used three types of samples. The samples of composition $\mathrm{Fe}_{84} \mathrm{~B}_{16}, \mathrm{Fe}_{40} \mathrm{Ni}_{40} \mathrm{Si}_{6} \mathrm{~B}_{14}$ and $\mathrm{Fe}_{82} \mathrm{~V}_{2} \mathrm{~B}_{16}$ were prepared by planar flow casting and tested for amorphicity in Wigner Research Centre for Physics, Budapest. The dimensions of samples were $105 \mathrm{~mm}$ long, 5 to $10 \mathrm{~mm}$ wide and about $0.030 \mathrm{~mm}$ thick. The magnetic parameters of the samples were determined by a computer-controlled magnetometer using two fluxgate type probes for measuring the stray field of the sample. The hysteresis loops were measured at room temperature under applying of stress in range from 0 to $20 \mathrm{MPa}$. The data obtained from descending branch of hysteresis loop were fitted by the formula $m=\sum_{k=0}^{n} A_{\mathrm{k}} \tanh \left(\alpha_{\mathrm{k}}\left(h-\mathrm{a}_{0 \mathrm{k}}\right)\right)$

Here $\mathrm{m}$ is the descending magnetization, $\mathrm{h}$ is the applied magnetic field, $A_{k}$ is the amplitude of the particular magnetization process, $\alpha_{\mathrm{k}}$ is the sheering factor and $\mathrm{a}_{0 \mathrm{k}}$ is the coercivity of the $\mathrm{k}$ 'th process $[5,6]$. The parameters were obtained by fitting the experimental descending branch using the least square fitting module of Origin software. From obtained fitting parameters the partial hysteresis loops were constructed.

\section{RESULTS AND DISCUSSIONS}

Fig. 1 shows the descending branches as a function of applied tensile stress. The inverse magnetoelastic effect is evident, the steepness of the branch increases as a function of applied stress.

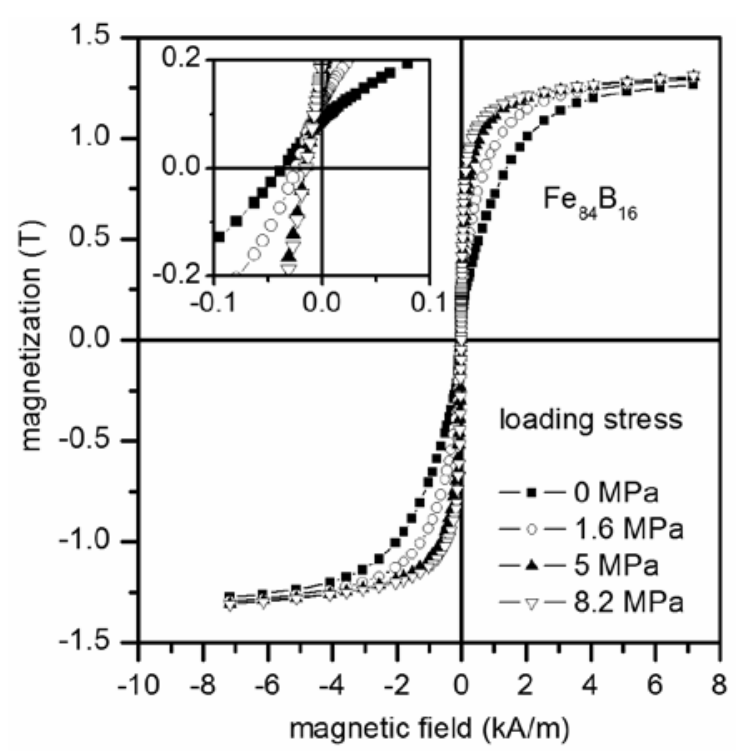

Fig. 1 Descending branch of measured hysteresis loops as a function of applied tensile stress 
We have found that 3 components fit better the experimental curve than 2 components as one can follow it in Fig. 2, where the fitting results for the non-stressed $\mathrm{Fe}_{84} \mathrm{~B}_{16}$ sample are presented.

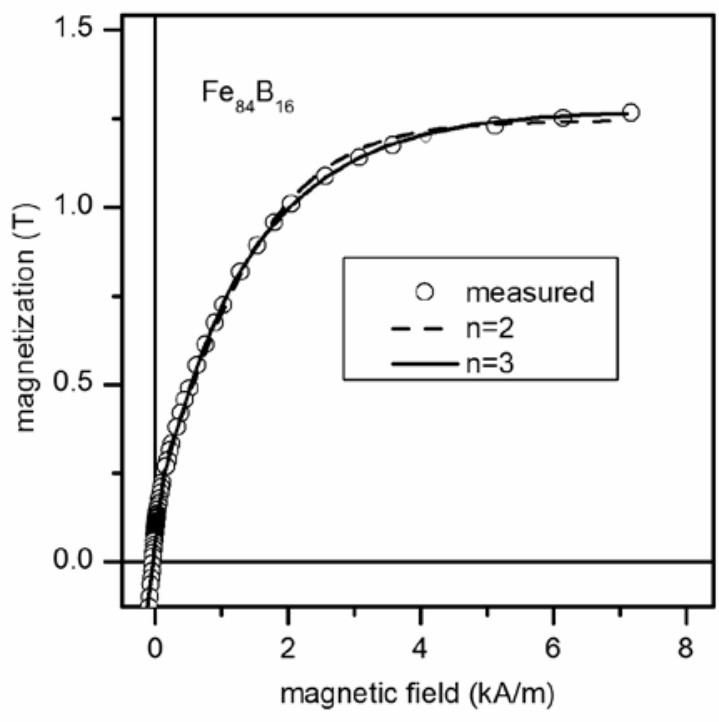

Fig. 2 The fitting quality obtained with two and three components

This is why, we have used for fitting calculations $n=3$ throughout this work taking into account all the three magnetization processes: DWM, DR and DWAN. A triplet of fitting parameters corresponds to each of these processes. In Figure 3 we present the original descending branch of the hysteresis loop together with the three constructed branches based on three triplets of fitting parameters.

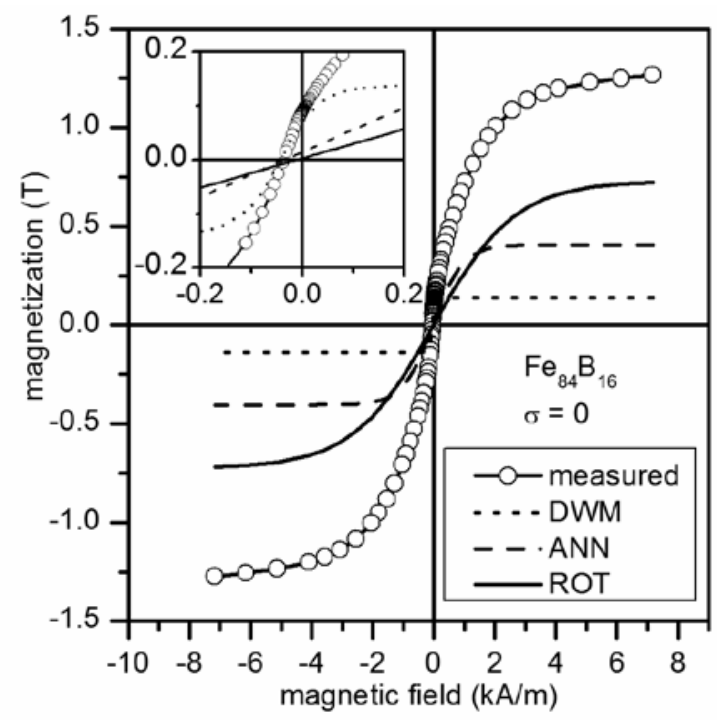

Fig. 3 Descending branches of the measured and fitted hysteresis loops

We assume that curve with the steepest increase to the saturation at relatively low magnetic fields represent the process of domain wall movement. The process of domain wall nucleation and annihilation is represented by the second steepest curve, saturating at a larger field. The
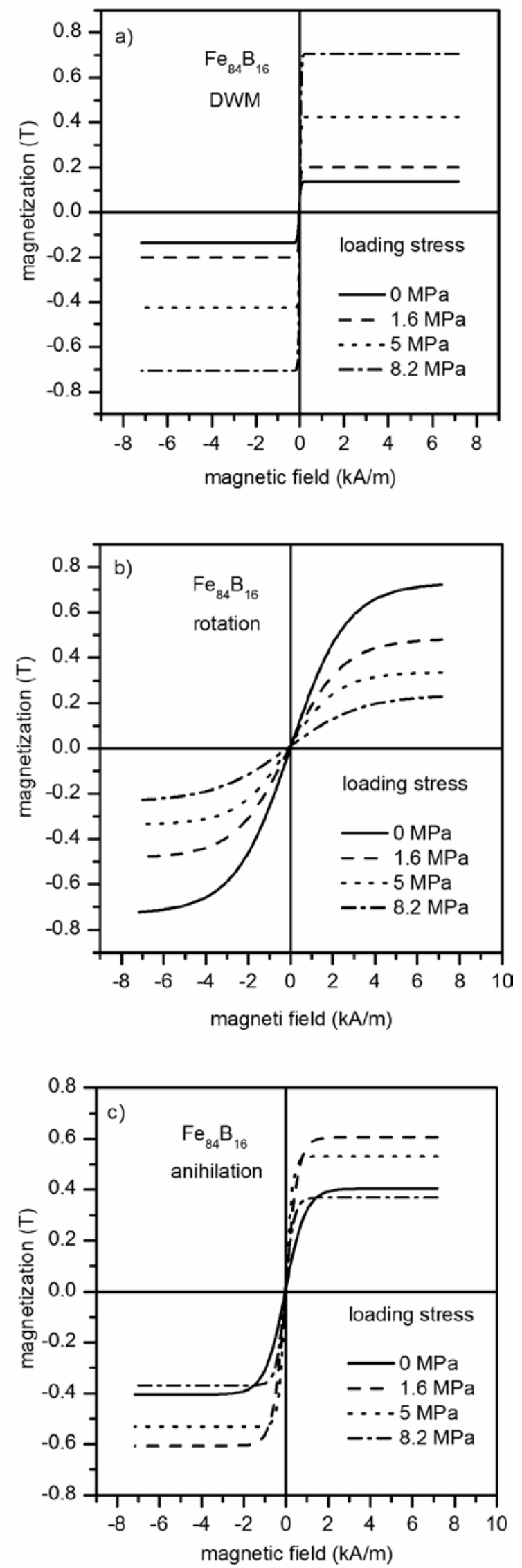

Fig. 4 Descending branches of the fitted hysteresis components at different applied stresses

curve corresponding to the domain rotation is the less steep and practically does not reach the saturation in 

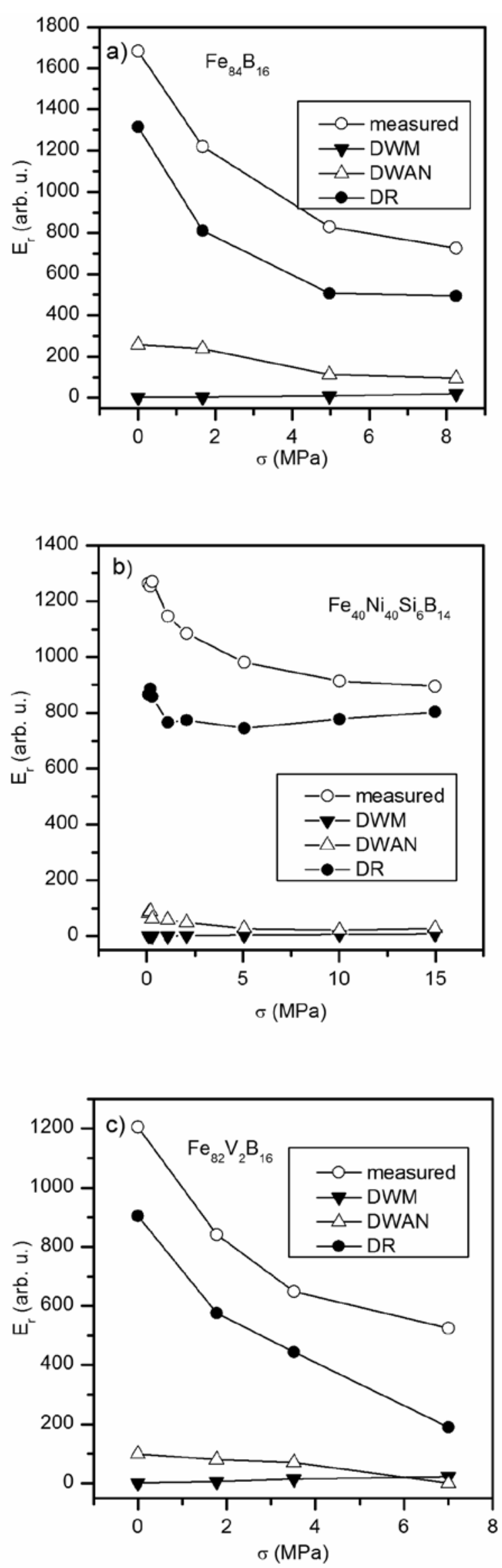

Fig. 5 The reversible energy of magnetization versus applied stress for different Fe-based metallic glasses

whole range of the applied magnetic fields. These components as a function of the applied tensile stress are represented in Fig 4a, 4b, and 4c. The continuous increase of the DWM component and decrease of DR component can be observed on Fig. $4 a$ and $4 b$. The DWAN component jumps to a maximal value for the smallest applied stress and then monotonously decreases with the stress. The behaviour of these components as a function of applied stress denotes the rearrangement of the domain structure along the stress induced anisotropy which is oriented along the ribbon axes for the positive magnetostrictive constant of the $\mathrm{Fe}_{84} \mathrm{~B}_{16}$ sample. For this longitudinal domain structure the role of the domain walls increases (both DWM and DWAN) and the contribution of the domain rotation decreases as one can follow the variation of the saturation values of the three components.

The change of the different contributions can be followed as well by the change of the reversible energy of magnetization, $E_{r}$, released at demagnetization process of the sample $[10,11]$. This $E_{r}$ can be calculated as the area between the vertical axes and the respective component curve taken from saturation down to the remanence value. It is worth to mention that this remanence value is not evidently visible at the scale of $\mathrm{kA} / \mathrm{m}$ used in Fig.4. This energy can be taken as a measure of the stress induced anisotropy.

The change of this energy, $E_{r}$ as a function of stress is very characteristic for different components as can be followed in Fig.5 for different Fe-based metallic glasses. Perusal of the figures $5 a, 5 b$ and $5 c$ reveals that out of the three components the domain rotation (DR) component is the most sensitive to the tensile stress. The other to components of the magnetization process (DWM and DWAN) are less sensitive, practically remains constant as a function of applied tensile stress.

This behaviour can be understood taking into account the stress induced anisotropy which has an easy axis along the ribbon length and increases linearly with the applied stress as $[12,13]$ :

$E_{s}=\frac{3}{2} \lambda_{s} \sigma \sin ^{2} \theta$

where $\theta$ represent the angle between the local $\mathrm{M}$ and the direction of the applied stress and $\lambda_{\mathrm{s}}$ is an isotropic magnetostriction coefficient. For samples with positive magnetostriction coefficient this induced anisotropy is along the ribbon axis and create domains parallel to the ribbon length. For such a longitudinal domain structure a more square-like hysteresis loop will form and the reversible energy of magnetization will decrease. Considering the three components, the two step-like DWM and DWAN components will show little area between the steep $\mathrm{M}(\mathrm{H})$ line and the vertical axis, which is hardly affected by the stress induced anisotropy, whereas the more flat DR component will show a large and decreasing reversible energy of magnetization as a function of $\sigma$. This fact justifies also the correct partition of the magnetization processes in the particular three processes.

\section{CONCLUSION}

Our study of the magnetization processes in FeB-based amorphous alloys showed that hyperbolic model is powerful tool for analysis of these processes in detail. It 
was clearly demonstrated the role of external stresses in the change of the partial contributions of the three components, DWM, DWAN and DR as a function of $\sigma$. The domain rotation component is mostly affected by the tensile stress, whereas the DWM and DWAN components are hardly affected in accordance with the role of the stress induced anisotropy.

\section{ACKNOWLEDGMENTS}

This work was performed within the frame of the project "New materials and technologies for energetics" ITMS: 26220220061, which is supported by the Operational Program "Research and Development" financed through European Regional Development Fund. This paper was developed as part of the project named "Centre of Excellence for Integrated Research \& Exploitation of Advanced Materials and Technologies in Automotive Electronics”, ITMS 26220120055.

\section{REFERENCES}

[1] PREISACH, F.: Über die magnetische Nachwirkung. Z. Phys. vol. 94, pp. 277-302, 1935.

[2] JILES, D. C. - ATHERTON, D. L.: Theory of ferromagnetic hysteresis, J. Appl. Phys. vol. 55, p. 2115, 1984.

[3] TAKACS, J.: Mathematics of Hysteretic Phenomena. Wiley-VCH. Berlin, 2003.

[4] JILES, D.: Introduction to Magnetism and Magnetic Materials. Loops for Sinusoidal and Distorted Waweforms. IEEE, Chapman \& Hall, London, 1998.

[5] VARGA, L. K. - KOVÁCS, Gy. - TAKACS, J.: Modeling the overlaping, simultaneous magnetization processes in ultrasoft nanocrystalline alloys, JMMM, vol. 320, pp. L26-L29, 2008.

[6] TAKACS, J. - KOVÁCS, Gy. - VARGA, L. K.: Decomposition of the hystersis loops of nanocrystalline alloys below and above the decoupling temperature, JMMM, vol. 320, pp. e1016-e1019, 2008.

[7] TAKACS, J.: A phenomenological mathematical model of hysteresis, Compel vol. 20, no. 4, pp. 10021004, 2001.

[8] VARGA, L. K. - KOVÁCS, Gy. - TAKACS, J.: Anhysteretic and bieased first magnetization curves for Finemet-type toroidal samples, JMMM , vol.320, pp. e814-e818, 2008.

[9] TAKACS, J. - KOVÁCS, Gy. - VARGA, L. K.: Hysteresis reversal, Physica B, vol. 403, 13-16, pp. 2293-3397, July 2008.

[10] KERSTEN, M.: Zur magnetischen Analyse der inneren Spannungen, Z. Phys. vol 76, pp.505, 1932.

[11] KERSTEN, M.: Zur magnetischen Analyse der inneren Spannungen II, Z. Phys. vol 82, pp.723, 1933.

[12] FIORILO, F.: Measurements and Characterization of Magnetic Materials, Amsterdam: Elsevier, 2004, p. 565.

[13] COEY, J. M. D.: Magnetism and Magnetic Materials, Cambridge: Cambridge University Press, 2009, p. 178.

Received November 13, 2012, accepted February 8, 2013

\section{BIOGRAPHY}

Jozef Kováč was born in 1954. In 1977 he graduated from the Faculty of Science, P. J. Šafárik University in Košice. He defended his CSc in 1989. Since 1977 he has been working at Institute of Experimental Physics Slovak Academy of Sciences, now as senior researcher in the Laboratory of Nanomaterials and Applied Magnetism. His research area is the study of magnetic properties of amorphous and nanocrystalline materials and nanoparticles. 